\title{
ACUTE DISTENSION OF THE GALL BLADDER IN CHILDREN
}

\author{
BY \\ WILLIAM RANKIN \\ From the Royal Hospital for Sick Children, Glasgow
}

(RECEIVED FOR PUBLICATION DECEMBER 10, 1954)

Diseases of the gall bladder in childhood are of great interest. Recently, Forshall and Rickham (1954) and previously Potter (1938) drew attention to cholecystitis associated with gall stones. The majority of such cases do not present as acute emergencies. Fèvre (1933) states that diseases of the biliary tract presenting as surgical emergencies are usually a perforation or volvulus of the gall bladder. There is, however, a third lesion which he has encountered, namely, acute distension of the gall bladder. This paper reports two such cases and supports views already held by French surgeons.

\section{Case Reports}

Case 1. A 4-year-old boy had been well until the day before admission. He then developed severe abdominal pain, intermittent in character, and suffered from nausea with occasional vomiting. The bowel movements had been normal but his urine was stated to have been dark. The pains lasted for some hours and then subsided, but soon returned and were apparently very severe though some relief was obtained by flexing the trunk. The pain was not referred to the gall bladder region but to the central abdomen. He was admitted to hospital with the tentative diagnosis of acute appendicitis.

He had complained of mild central abdominal pain for six months, but in general his health was good.

On admission the temperature was $99^{\circ} \mathrm{F}$., pulse rate 132 , and respiratory rate 24 per minute. The child lay quietly and was not in severe pain. The face was flushed, the throat injected, skin and sclerae were clear. There was marked tenderness of the right hypochondrium and epigastrium. Tenderness was elicited in the right iliac fossa but here there was no rigidity. It was possible to feel the edge of the liver but there was no local swelling nor was any mass palpable in the abdominal cavity. Bowel sounds were present in all areas. Rectal examination was negative. A rectal wash-out revealed pale faecal material, some mucus, and a fleck of red blood. Bile was not present in the urine.

Shortly afterwards the child had a severe attack of pain; he vomited profusely, and there was marked rigidity in the right hypochondrium. A tentative diagnosis of intussusception was made and the child prepared for operation.

Under anaesthesia a tense mass was palpable in the right hypochondrium. On opening the abdomen clear fluid escaped. Numerous enlarged, injected and firm lymph nodes were palpable in the mesentery and duodeno-jejunal flexure. The caecum was located, the appendix was not inflamed but was removed. There was no abnormality in the small intestine and the large gut was traced uneventfully to the rectum. On displacing the transverse colon downwards, a large, tense gall bladder presented. Owing to extreme distention, it was not possible to determine the presence or absence of stones in the viscus but none were found in the cystic duct or common ducts neither of which was dilated. The gall bladder was essentially normal in colour; there was no thickening or obvious signs of infection. Further pressure did not empty it. Because of the distension packs were inserted, and the operative field was prepared as for cholecystectomy. As the organ was so normal apart from its distension, it was decided that aspiration would suffice and $40 \mathrm{ml}$. of dark green bile were aspirated. The needle was removed and the puncture sealed with a purse string suture. The abdomen was closed without drainage.

Apart from mild pyrexia for $\mathbf{4 8}$ hours convalescence was uneventful. When reviewed four months later the patient was well. Pain had not recurred.

Case 2. A 10-year-old boy had been well until four days before admission. He complained of severe abdominal pain, umbilical in distribution. His appetite had been poor, he had had some nausea, but had not vomited. As the pain subsided, he went to school the next morning, but was sent home in the afternoon owing to a recurrence of abdominal symptoms. At home his doctor noted increased tonicity of the right rectus muscle, particularly in its upper half. Tenderness was elicited over the distribution of the small bowel mesentery. The condition again subsided until the afternoon of the day of admission, when the child was nauseated, and severe pain had recurred. The right rectus muscle was in acute spasm and there was marked rigidity in the right hypochondrium. During transit to hospital by car, over a route paved with cobblestones, the child com- 
plained bitterly of the pain, which was accentuated by the jolting.

Before his illness the child had been well, but his appetite had been variable and he was fussy about food, not liking eggs and fat. He had received treatment for a threadworm infestation.

On admission the temperature was $98^{\circ} \mathrm{F}$., pulse rate 96 and respiratory rate 22 per minute. The child lay quietly in bed. The face was pale and the fauces were clear, as were the skin and sclerae. There was marked tenderness and rigidity in the right hypochondrium. The child could point to the site of maximum tenderness in the subhepatic region, $2 \frac{1}{2}$ in. above McBurney's point. He resented palpation in the area. He was seen by the senior surgeon who made a pre-operative diagnosis of inflammation of a high-placed appendix and cholecystitis. In view of the definite rigidity, which could not be ignored, the child was prepared for the theatre.

At operation by Mr. Matthew White, through a right rectus incision the appendix was located in the right iliac fossa. It was injected but not acutely inflamed and was removed. Numerous mesenteric nodes were present. The gall bladder was tensely distended, and it was obviously the cause of the abdominal tenderness. The gall bladder was normal in colour and appeared slightly thickened. The remarkable tension within the viscus prevented excluding the presence or absence of a calculus. The lymph nodes in the region of the cystic duct was palpable, firm and enlarged. Some doubt arose as to whether one was indeed a node or a stone impacted in the cystic duct. The common bile duct was not dilated and so consequently $30 \mathrm{ml}$. of bile was aspirated. The bile was green and contained mucus. A cholecystostomy was performed, no stones were found, and the abdomen was closed.

Section of the appendix showed a mild acute catarrhal reaction, and some tomato pips in its lumen. Culture of the bile was sterile.

For the first four post-operative days the child ran a temperature of 99 to $101^{\circ} \mathrm{F}$. Seven ounces of bile were drained in the first 24 hours. On the fourth day the urine was dark but no bile was present. No icterus occurred but a transitory pruritis of the chest wall. The bowels moved on the fifth day and were normal in colour and contained bile pigment. Thereafter recovery was uneventful and the patient was discharged on the sixteenth post-operative day. One month later threadworms were found in a bowel wash-out.

\section{Discussion}

These two cases were acute surgical emergencies and were not associated with stone formation. It is emphasized that in such cases the gall bladder is not only tense but is grossly distended. In both cases $30 \mathrm{ml}$. of bile were aspirated and despite this the gall bladder was by no means emptied. Following aspiration the gall bladder was still larger than usual.

The pathology of the condition is obscure; a biliary dyskinesia could be postulated, but the condition appears to be the result of an inflammatory reaction of the cystic lymph node. In both cases there was an extensive and acute mesenteric adenitis. In the second case the cystic node was palpable. Due to unfamiliarity with this condition, an accurate assessment of the node was not made in Case 1 . The cystic duct in both cases appeared to be normal but a larger lymph node could have obstructed the cystic duct and a situation might have arisen as described by Lee and Englender (1953) in which a hydrops of the gall bladder was found which actually caused a pyloric obstruction; this case had an ascaris infestation. In Case 2 a known threadworm infestation was being treated.

The pain in these cases is severe and can simulate a gall bladder colic. The child has relief for some time and then the pain recurs. The rigidity of the rectus muscle is marked and from its site and intensity may suggest an underlying perforation. The French writers have commented upon this feature. The pre-operative diagnosis is infection of a highly situated appendix or intussusception. Acute cholecystitis may be considered. Ombrédanne (1946) states bluntly that such cases are "too easily diagnosed as appendicitis'. The cases presented similarly to those described by Fèvre (1933) and at operation almost identical findings were observed.

Discussion will always arise as to what operative procedure should be carried out when confronted with this condition. Such cases are usually met with by the younger surgeon. It is recommended that simple aspiration of the gall bladder will suffice. This procedure was effective in my first case, and was.the method chosen by Fèvre in his two cases. Cholecystostomy should be reserved for those cases in which there is doubt as to whether the bile is infected or in which there is a possible associated cholelithiasis. The observations of Edington (1925, 1930) suggest that in more experienced hands the latter complication should be dealt with by cholecystectomy. Where gross pathological changes in the gall bladder and cystic duct are noted cholecystectomy is deemed advisable.

My findings in this condition are in accordance with and support the opinions of the French surgeons who drew attention to this interesting clinical entity.

My thanks are due to Mr. A. P. Laird, under whose care the patients were admitted, for permission to publish these cases, and to Mr. Mattnew Wnite whom I assisted in the second case.

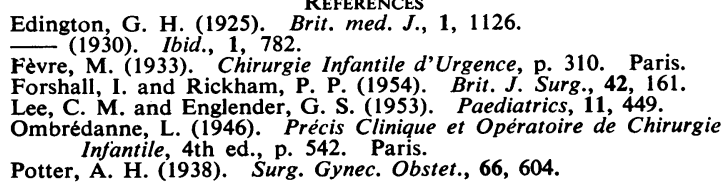

